\title{
A Computational Approach on the Activity of Hesperidin as Antagonist for Proteins of SARS-CoV-2
} \author{
Muralidhara Rao Dowlatabad 1, * (D)

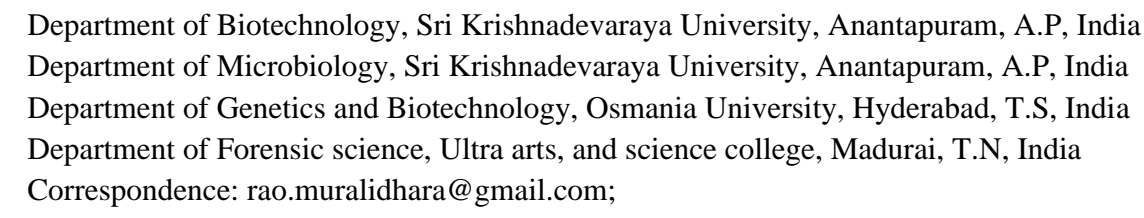

Satyanarayana Swamy Vyshnava 1D, Dileep Kumar Kanderi 2(D), Shiva Prasad Panjala ${ }^{3}$, Kameshpandian Paramasivam 4 (D), Gayathri Pandluru 1, Swathi Banapuram ${ }^{3}$, Roja Rani Anupalli ${ }^{3}$,

Received: 21.12.2020; Revised: 10.01.2021; Accepted: 12.01.2021; Published: 20.01.2021

\begin{abstract}
Spike proteins are a functional component in the viral infections of the SARS-CoV-2 virus that binds to the ACE2 receptors of the human cells. The naturally derived drugs like hesperidin show a higher affinity to avoiding the binding of the spike protein. Inhibition of the spike protein study represents the development of a new drug for SARS disease. Along with the hesperidin and its analogs have been found effective medicine to control SARS-CoV-2; the entire drug bank database was screened for strong analogous remedy like compounds as hesperidin. Virtual Screening and docking studies were intended for these molecules against spike protein with auto dock virtual screening tool and auto dock vena. The docking outcome showed that the compounds hesperitin, silibilin, and dihydromyricetin were having the highest binding energies, like -8 and -6.2. The current study indicates that the lead molecules have to be evaluated to improve prospective drug molecules.
\end{abstract}

Keywords: computational drug docking; hesperidin; spike protein; SARS-CoV-2.

(c) 2020 by the authors. This article is an open-access article distributed under the terms and conditions of the Creative Commons Attribution (CC BY) license (https://creativecommons.org/licenses/by/4.0/).

\section{Introduction}

A health emergency and rapid infection transmission rate were created by the amid an outbreak of global pandemic COVID-19. There is a spent argument on the origin of the virus, where it comes from and affecting almost all countries levels of spread and severity of the certain alarming levels as mention by WHO, characterized as a pandemic.

The novel corona is creating a panic situation worldwide due to inadequate risk assessment, research, and treatment regarding the pandemic's urgency. Due to increased globalization, intra-regional and country travels to make the global epidemics threaten public health, with a very little preventive measure and antiviral therapies revealing viral infections remained only with few antiviral drugs adopted for medical practice.

Naturally occurring large groups of biochemicals such as terpenoids, flavonoids, phenolic compounds, etc., from the plant sources, may look at potential drug molecules to treat the deadly diseases [1]. One such compound hesperidin, which is a glycosidic phenolic flavonoid compound and it is abundant in citric fruits such as lemons, grapefruits, etc., This flavonoid shows anti-inflammatory, anti-fungal, antibacterial, antiviral, antioxidant, and free radical scavengers against many pathogenic and immunomodulatory activities [2-4]. The 
molecular studies and docking studies refer to the potential application of hesperidin in treating microbial infections [4], neurological disorders like Parkinson and Alzheimer's disease [5,6], anti-inflammatory activity [7], anti-cancer activity [8], and other in-silico studies of the modified to nanoparticles in the application of antioxidant activity [9].

In-silico studies in identifying the antiviral drugs against COVID-19 are processed in recent literature, where hesperidin is one of its kind shows the potential application in inhibiting the protease [10-12] and other related proteins of the Coronavirus 2 [13]. After considering the potential application of hesperidin, in this study, we have found the antiviral activity of hesperidin and its derivatives as listed in the drug bank using the in-silico analysis.

\section{Materials and Methods}

\subsection{Protein preparation.}

The target protein crystal structure, the spike core protein, was acquired from the protein data bank with pdb id1WNC along with other target proteins include1QZ8, 6W4Band 3EE7 (which are Nsp9 RNA binding proteins structures), and 6W63 (main protease) [14]. Using the discovery studio client, the targeted proteins' molecular coordinates were estranged, and its geometry was optimized for the ligand interactions during the docking workplace in .pdbqt format [15].

\subsection{Binding site prediction.}

Finding molecular coordinates is the most critical step in molecular docking in the modern era of drug design. A large number of online algorithms are available to find the active sites for the selected target proteins; in this study, to find the active site for the respective receptors, we acknowledged using the online platform playmolecule_Deep Site server [16]. This program, playmolecule_Deep Site, is used to locate and measure protein pockets and cavities mechanically and is based on accurate computational geometry methods and alpha form, and distinct flow theory.

\subsection{Ligand preparation.}

In this study, plant-based flavanone glycoside hesperidin, abundant in citrus fruits, was used to study the activity against the SARS-COV-2. We acquired the requisite files (.sdf and .pdb) from the drug bank with ID DB04703 for hesperidin. With hesperidin compound, its potential analogs were used in an assay for the binding on the viral in targeted proteins [17]. Using auto dock tools 4.0, the ligands are optimized for docking by creating the atomic coordinates with .pdbqt formats [18].

\subsection{Virtual screening.}

In this study, the most powerful yet reliable platform, Auto dock 4.0, was implemented for the virtual screening of the hesperidin and its analogs to the targeted proteins of COVID19. Based on the standard operations' Auto dock execution, provide a list of outcomes to be ordered in the rank based on the affinity-based grading system. 


\subsection{Structural analysis and visualization.}

The powerful tools represent analysis and illustration of the protein-ligand interactions include PyMol from Schrödinger [19] and Discovery studio client from the Dassault Systèmes [15].

\section{Results and Discussion}

Playmolecule_Deep Site server provides the possible coordinates for the active site for the respective protein molecules, where $1 \mathrm{WNC}$ has core three active sites with active amino acids include THR 921, GLY 928, ASP932, GLN 931, LYS 946, GLN 1161, ASN 1168 involves in considerably in binding and catalytic activity. The Virtual screening of the ligands in Fig 1 includes hesperidin and its structural analogs Hesperetin, Taxifolin, Sakuranetin, Silibinin and Dihydromyricetin were capable approach in potential inhibitors in the ACE 2 (Angiotensin 2 Receptor) binding in the SARS COV2 virus based on the values obtained in the order $-8,-5.7,-6.1,-5.3$ and $-6.2 \mathrm{Kcal} / \mathrm{Mol}$.

When comparing the other protein structure include Nsp9 RNA binding proteins structures of positive-strand of SARS COV2 virus with PDB IDs 1QZ8, 6W4B and 3EE7, 3EE7 had better binding affinities than the other Nsp9 binding protein. This may occur due to the available active site amino acids' potential to form the higher affinity binding in the 3EE7 rather than that of other Nsp9 Proteins based on this docking study. The binding of the ligand on the targeted Nsp9 protein suggests that hesperidin and its analogs have a considerable function in the self-assembly of the proteins during the replication of the virus for the RNA binding, which may provide an effective drug model for the treatment of COVID-19 patients to stop the intracellular transmission of the viruses.

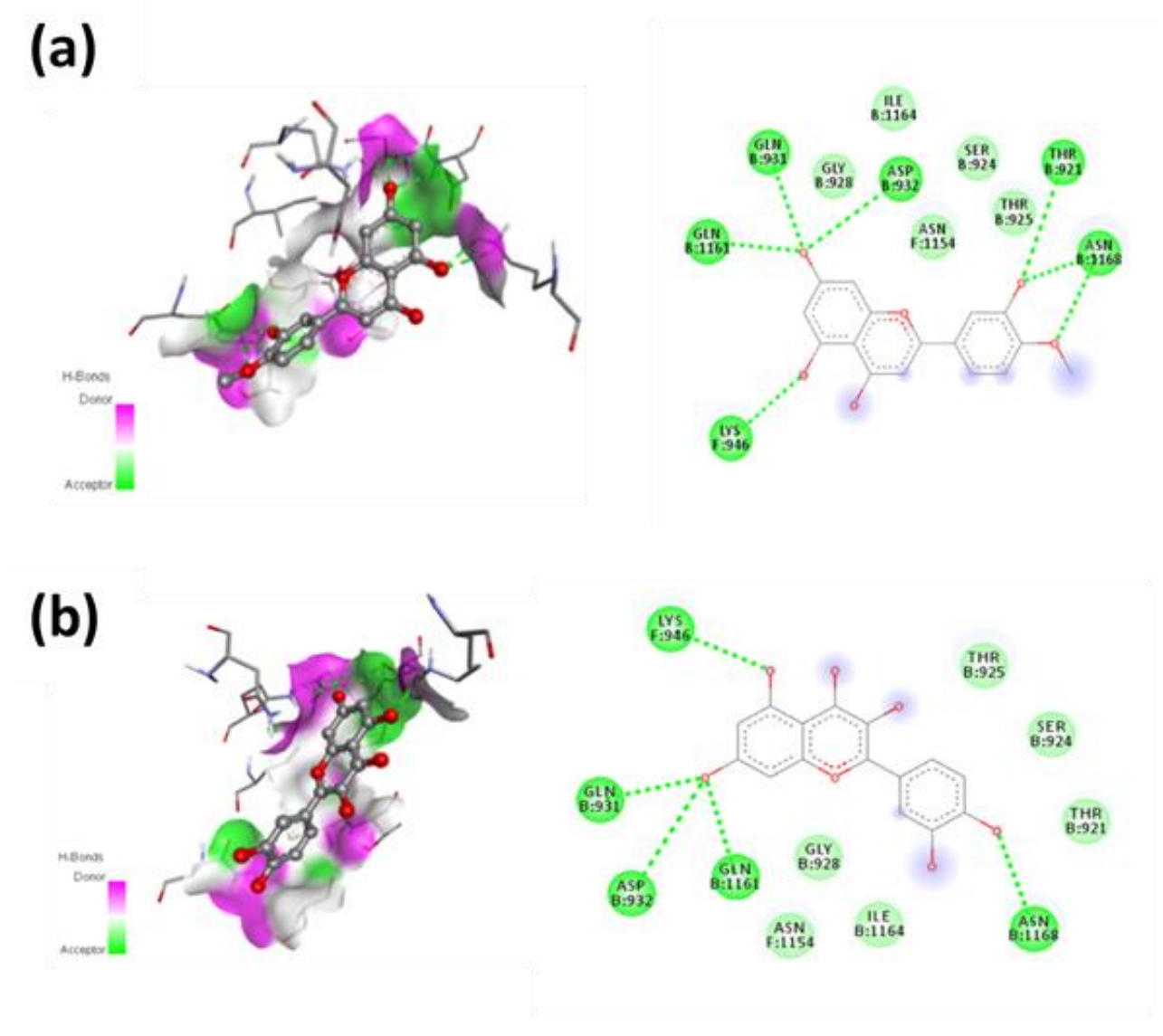



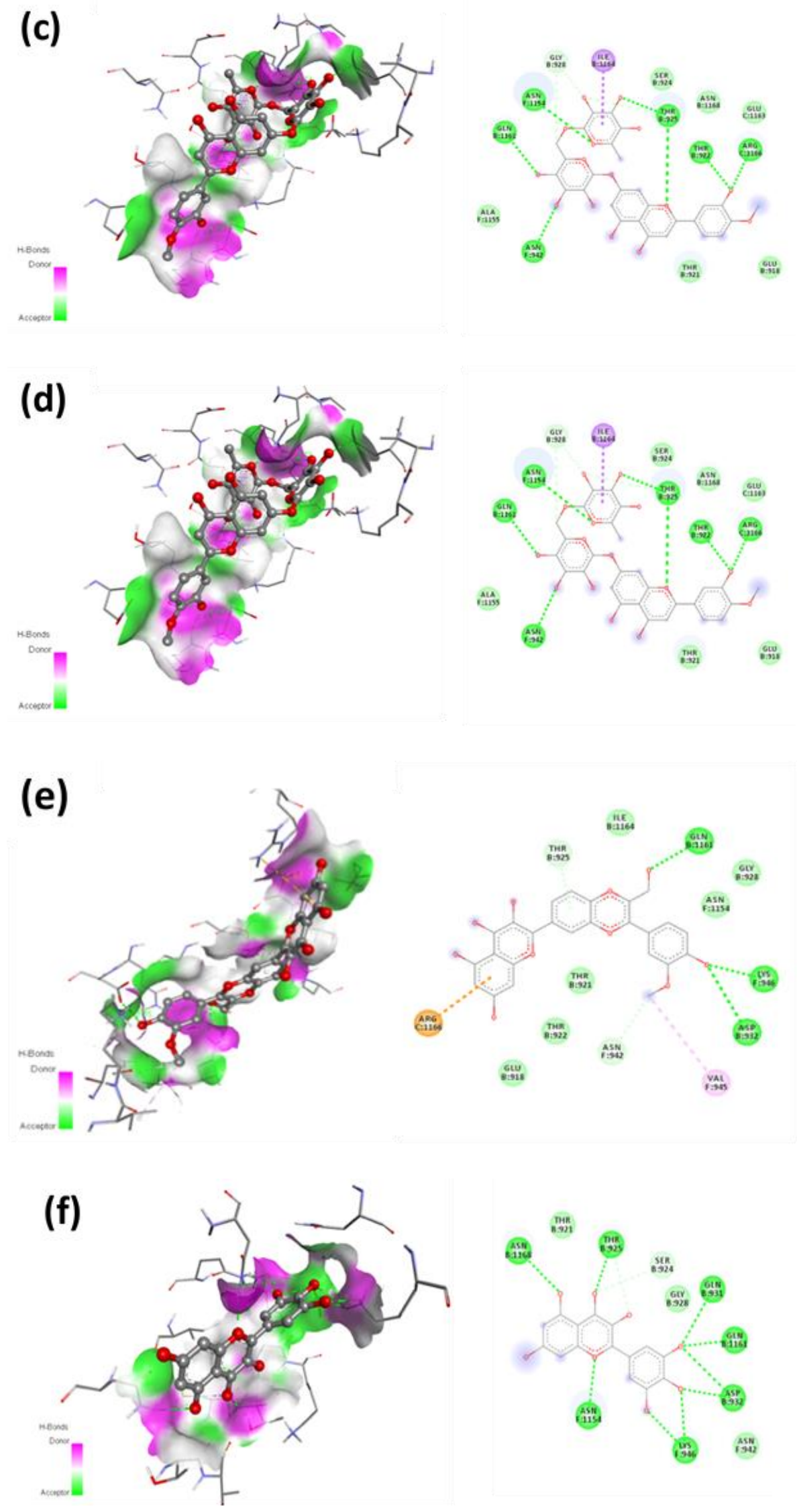

Figure 1. Molecular docking of the $1 \mathrm{WNC}$, the spike protein core structure, had profound activity in the binding on the angiotensin receptor 2 (ACE 2). (a) Hesperetin with -8.0 Kcal/Mol, (b) Taxifolin with -5.7 Kcal/Mol, (c) Sakuranetin with -6.1 Kcal/Mol, (d) Silibininwith -5.3 Kcal/Mol, and (e) Dihydromyricetin with -6.2 Kcal/Mol affinities for arbitrary activities include inhibition and inactivation of the protein. 
Protease, also called the main protease, is the potential source of infections of SARSCOV-2 viruses. The inhibitory activity was well studied and understood from the recent investigations by in-silico analysis states the enzyme inactivation and blocking the cellular receptor for the protease activity [12]. Based on this source, our targeted main protease protein with PDB ID 6W63 had reproduced similar results with hesperidin analogs with values ranges from -5.4 for hesperetin, -5.6 for dihydromyricetin, and -5.8 for taxifolin.

The virtual screening results for the targeted protein with respective ligands for the presented study are listed and compared in Fig 2 (detailed discussion is elaborated in Table 1), where for the overall study, the highest binding affinity was higher with $-8.0 \mathrm{Kcal} / \mathrm{Mol}$ in the $1 \mathrm{WNC}$ with hesperidin molecule. 1WNC spike core protein variance in the amino acid residual functions are studied using the molecular simulations with references to the RMSF with minimal changes in the residual fluctuations, which refers to the binding affinity for respective ligands are shown in Fig 3. This shows the functional activity of $1 \mathrm{WNC}$ during its bindings to the respective ligands include hesperidin and its structural analogs.

Among the docked ligands of hesperidin and its analogs on the Nsp9 protein, main protease and Spike core protein had a very higher tendency, which may have arbitrary effects include inhibition of the protein to bind the ACE 2 receptor on the COVID-19 patients to block the infections of the SARS COV2 virus. To understand the core activities for the present study of protein-ligand interactions, wet lab analysis may provide deep insights into the application of this flavonoid and its analogs in treating this current pandemic.

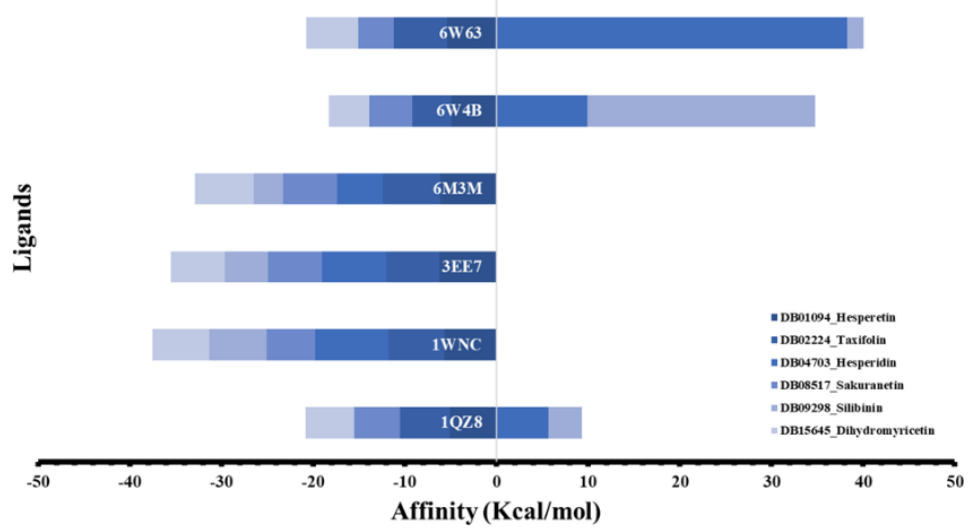

Figure 2. Molecular docking of the 1WNC, 1QZ8, 3EE7, 6M3M, 6W4B, and 6W63 protein structures affinities for arbitrary activities include inhibition and inactivation of the protein, where $1 \mathrm{WNC}$ has a higher affinity towards the hesperidin.

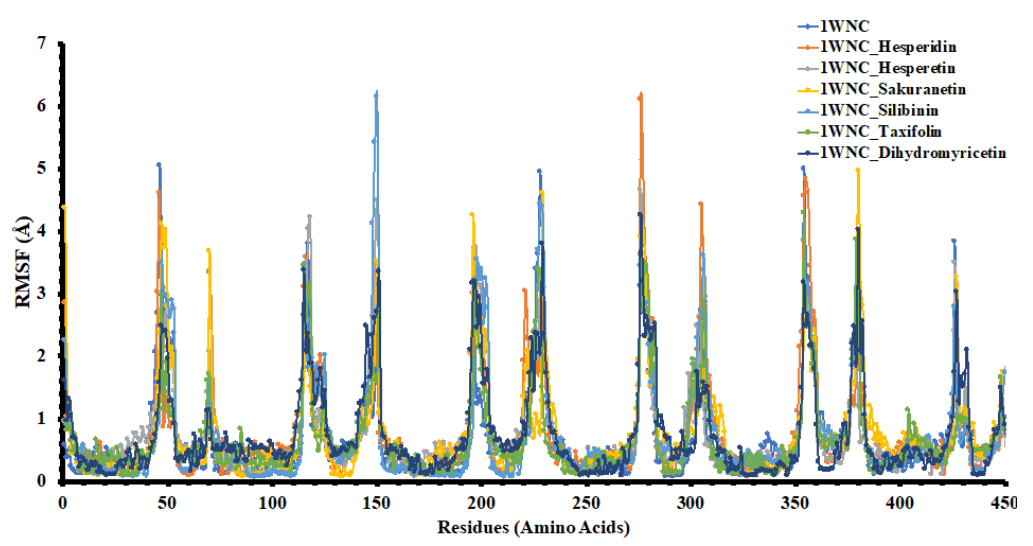

Figure 3. Molecular dynamics studies of the $1 \mathrm{WNC}$, the spike protein core structure which had profound activity in the binding on the angiotensin receptor 2 (ACE 2) RMSF with minimal changes in the residual fluctuations, which refers to the binding affinity for respective ligands. 
Table 1. Molecular docking of the 1WNC, 1QZ8, 3EE7, 6M3M, 6W4B, and 6W63 protein structures affinities for arbitrary activities include inhibition and inactivation of the protein.

\begin{tabular}{l|c|c|c|c|c|c} 
Ligands & $\mathbf{1 Q Z 8}$ & $\mathbf{1 W N C}$ & $\mathbf{3 E E 7}$ & $\mathbf{6 M 3 M}$ & $\mathbf{6 W 4 B}$ & $\mathbf{6 W 6 3}$ \\
\hline DB01094_Hesperetin & -5.1 & $\mathbf{- 5 . 7}$ & -6.2 & -6.1 & -4.9 & -5.4 \\
\hline DB02224_Taxifolin & -5.4 & $\mathbf{- 6 . 1}$ & -5.8 & -6.3 & -4.3 & -5.8 \\
\hline DB04703_Hesperidin & 5.7 & $\mathbf{- 8 . 0}$ & $\mathbf{- 7 . 0}$ & -5 & 9.9 & 38.3 \\
\hline DB08517_Sakuranetin & -5 & $\mathbf{- 5 . 3}$ & -5.9 & -5.9 & -4.7 & -3.9 \\
\hline DB09298_Silibinin & 3.6 & $\mathbf{- 6 . 2}$ & -4.7 & -3.2 & 24.9 & 1.8 \\
\hline DB15645_Dihydromyricetin & -5.3 & $\mathbf{- 6 . 2}$ & -5.9 & -6.4 & -4.4 & -5.6
\end{tabular}

\section{Conclusions}

The current pandemic situation created an emergency state that is looking for a potential anti virtual drug for the COVID-19 treatment. Our efforts were kept in this study to determine the activity of the natural products such as hesperidin. A flavonoid compound in the citrus fruit may help treat the SARS-CoV2 infection by inhibiting the protein such as protease, Nsp9 RNA binding protein, and Spike protein through an in-silico study. Our study provides a promising application of hesperidin and its analogs. We expect this study to be supportive in designing novel and effective inhibitors against COVID-19 and other severe acute respiratory diseases.

\section{Funding}

This research received no external funding.

\section{Acknowledgments}

This work was motivated by the present pandemic situation to handle humanity from the COVID-19.

\section{Conflicts of Interest}

The authors declare no conflict of interest.

\section{References}

1. Bergman, M.M. The World after COVID. Multidisciplinary Digital Publishing Institute, 2020; https://doi.org/10.3390/world1010005

2. Yilmaz, M.M.; Szabolcs, M.J.; Geskin, L.J.; Niedt, G.W. An Autopsy Review:" COVID Toes". The American Journal of Dermatopathology 2020, https://doi.org/10.1097/dad.0000000000001827.

3. Alsulami, A.F.; Thomas, S.E.; Jamasb, A.R.; Beaudoin, C.A.; Moghul, I.; Bannerman, B.; Copoiu, L.; Vedithi, S.C.; Torres, P.; Blundell, T.L. SARS-CoV-2 3D database: understanding the coronavirus proteome and evaluating possible drug targets. Briefings in Bioinformatics 2021, https://doi.org/10.17863/CAM.62145.

4. Yan, Y.; Shen, X.; Cao, Y.; Zhang, J.; Wang, Y.; Cheng, Y. Discovery of Anti-2019-nCoV Agents from 38 Chinese Patent Drugs toward Respiratory Diseases via Docking Screening. 2020; https://doi.org/10.20944/preprints202002.0254.v2.

5. Garg, A.; Garg, S.; Zaneveld, L.J.; Singla, A.K. Chemistry and pharmacology of the Citrus bioflavonoid hesperidin. Phytotherapy research : PTR 2001, 15, 655-669.

6. Wacker, A.; Eilmes, H.G. [Antiviral activity of plant components. 1st communication: Flavonoids (author's transl)]. Arzneimittel-Forschung 1978, 28, 347-350.

7. Goris, T.; Pérez-Valero, Á.; Martínez, I.; Yi, D.; Fernández-Calleja, L.; San León, D.; Bornscheuer, U.T.; Magadán-Corpas, P.; Lombó, F.; Nogales, J. Repositioning microbial biotechnology against COVID-19: the case of microbial production of flavonoids. Microbial Biotechnology 2020, https://doi.org/10.1111/17517915.13675.

8. Kaur, G.; Tirkey, N.; Chopra, K. Beneficial effect of hesperidin on lipopolysaccharide-induced hepatotoxicity. Toxicology 2006, 226, 152-160, https://doi.org/10.1016/j.tox.2006.06.018. 
9. Al-Ashaal, H.A.; El-Sheltawy, S.T. Antioxidant capacity of hesperidin from Citrus peel using electron spin resonance and cytotoxic activity against human carcinoma cell lines. Pharmaceutical Biology 2011, 49, 276282, https://doi.org/10.3109/13880209.2010.509734.

10. Iranshahi, M.; Rezaee, R.; Parhiz, H.; Roohbakhsh, A.; Soltani, F. Protective effects of flavonoids against microbes and toxins: The cases of hesperidin and hesperetin. Life Sciences 2015, 137, 125-132, https://doi.org/10.1016/j.lfs.2015.07.014.

11. Yu, R.; Chen, L.; Lan, R.; Shen, R.; Li, P. Computational screening of antagonists against the SARS-CoV2 (COVID-19) coronavirus by molecular docking. International Journal of Antimicrobial Agents 2020, 56, https://doi.org/10.1016/j.ijantimicag.2020.106012.

12. Nagappan, P.; Krishnamurthy, V. Structural prediction and comparative molecular docking studies of hesperidin and L-Dopa on A-Synuclein, MAO-B, COMT and UCHL-1 Inhibitors. International Journal of Pharmaceutical and Clinical Research 2015, 7, 221-225.

13. Chakraborty, S.; Bandyopadhyay, J.; Chakraborty, S.; Basu, S. Multi-target screening mines hesperidin as a multi-potent inhibitor: Implication in Alzheimer's disease therapeutics. European Journal of Medicinal Chemistry 2016, 121, 810-822, https://doi.org/10.1016/j.ejmech.2016.03.057.

14. Rehman, K.; Munawar, S.M.; Akash, M.S.H.; Buabeid, M.A.; Chohan, T.A.; Tariq, M.; Jabeen, K.; Arafa, E.-S.A. Hesperidin improves insulin resistance via down-regulation of inflammatory responses: Biochemical analysis and in silico validation. PLOS ONE 2020, 15, https://doi.org/10.1371/journal.pone.0227637.

15. Al-Shmgani, H.; Shakir, R.; Wahed, A.; Elekofehinti, O. Design, Synthesis, Docking, Antitumor Screening, And Absorption, Distribution, Metabolism, And Excretion Prediction Of New Hesperdin Derivative. Asian Journal of Pharmaceutical and Clinical Research 2020, 13, 24-31, https://doi.org/10.22159/ajpcr.2020.v13i1.35684.

16. Praveen Kumar, P.; Sunil kumar, K.T.; Kavya Nainita, M.; Sai Tarun, A.; Raghu Ramudu, B.G.; Deepika, K.; Pramoda, A.; Yasmeen, C. Cerebroprotective Potential of Hesperidin Nanoparticles Against Bilateral Common Carotid Artery Occlusion Reperfusion Injury in Rats and In silico Approaches. Neurotoxicity Research 2020, 37, 264-274, https://doi.org/10.1007/s12640-019-00098-8.

17. Mohamed, K.; Yazdanpanah, N.; Saghazadeh, A.; Rezaei, N. Computational drug discovery and repurposing for the treatment of COVID-19: A systematic review. Bioorganic chemistry 2021, 106, 104490-104490, https://doi.org/10.1016/j.bioorg.2020.104490.

18. Adem, S.; Eyupoglu, V.; Sarfraz, I.; Rasul, A.; Ali, M. Identification of potent COVID-19 main protease (Mpro) inhibitors from natural polyphenols: An in silico strategy unveils a hope against CORONA. 2020, https://doi.org/10.20944/preprints202003.0333.v1.

19. Ganeshpurkar, A.; Saluja, A., Immunomodulatory effect of rutin, catechin, and hesperidin on macrophage function. Indian Journal of Biochemistry and Biophysics 2020, 57, 58-63.

20. Pagliaro, M.; Meneguzzo, F. Hydroxychloroquine for the Treatment of Coronavirus Disease 2019: Evidence, Possible Mode of Action and Industrial SupplyHydroxychloroquine for the Treatment of Coronavirus Disease 2019: Evidence, Possible Mode of Action and Industrial Supply. Medicine Research 2020, 4, https://doi.org/10.21127/yaoyimr20200014.

21. Topçu, G.; Altan, V.M.; Alim Toraman, G.Ö.; Şenol, H. Natural Alkaloids as Potential Anti-Coronavirus Compounds. 2020.

22. Berman, H.M.; Westbrook, J.; Feng, Z.; Gilliland, G.; Bhat, T.N.; Weissig, H.; Shindyalov, I.N.; Bourne, P.E. The Protein Data Bank. Nucleic Acids Res 2000, 28, 235-242, https://doi.org/10.1093/nar/28.1.235.

23. Biovia, D.S. Discovery studio. Dassault Systèmes BIOVIA. 2016.

24. Jiménez, J.; Doerr, S.; Martínez-Rosell, G.; Rose, A.S.; De Fabritiis, G. DeepSite: protein-binding site predictor using 3D-convolutional neural networks. Bioinformatics 2017, 33, 3036-3042, https://doi.org/10.1093/bioinformatics/btx350.

25. Wishart, D.S.; Knox, C.; Guo, A.C.; Shrivastava, S.; Hassanali, M.; Stothard, P.; Chang, Z.; Woolsey, J. DrugBank: a comprehensive resource for in silico drug discovery and exploration. Nucleic Acids Res 2006, 34, D668-D672, https://doi.org/10.1093/nar/gkj067.

26. Morris, G.M.; Goodsell, D.S.; Halliday, R.S.; Huey, R.; Hart, W.E.; Belew, R.K.; Olson, A.J. Automated docking using a Lamarckian genetic algorithm and an empirical binding free energy function. Journal of Computational Chemistry 1998, 19, 1639-1662, https://doi.org/10.1002/(SICI)1096987X(19981115)19:14<1639::AID-JCC10>3.0.CO;2-B.

27. Schrödinger Release 2020-4: BioLuminate, Schrödinger, LLC, New York, NY, 2020. 